\title{
Heparin-Induced Thrombocytopenia Presenting as Splenic Hemorrhage Following Cardiac Surgery: A Case Report
}

\author{
J Ferry ${ }^{1 *}, S$ Youssef ${ }^{2}, P W U^{2}$ and $L$ Hegerova ${ }^{3}$ \\ ${ }^{1}$ Department of Hematology and Oncology, Swedish Medical Center, Seattle, WA, USA \\ ${ }^{2}$ Swedish Heart and Vascular - Cardiac Surgery, Seattle, WA, USA \\ ${ }^{3}$ Swedish Center for Blood Disorders and Stem Cell Transplantation, Seattle, WA, USA
}

*Corresponding author: Joseph Ferry, Department of Hematology and Oncology, Swedish Medical Center, Seattle, WA, USA

\section{Essentials \\ - HIT-associated antibodies are frequently detected after cardiac surgery. \\ - We report a case of atraumatic splenic hemorrhage due to HIT following coronary artery bypass grafting. \\ - HIT should be suspected for any patient with congestive hem- orrhage following venous thrombosis, thrombocytopenia and recent heparin exposure. \\ - Hemorrhagic splenic infarction due to splenic vein thrombosis is reminiscent of bilateral adrenal hemorrhage (BAH) due to adrenal necrosis seen in HIT.}

\begin{abstract}
Heparin induced thrombocytopenia with thrombosis (HIT) is a paradoxical prothrombotic complication of anticoagulant therapy. We report a case of atraumatic splenic hemorrhage due to splenic vein thrombosis as main indicator to diagnosis of HIT. The presentation is reminiscent of the rare bilateral adrenal hemorrhage due to adrenal necrosis that occurs in HIT. Alternative anticoagulation is mainstay of therapy for HIT despite hemorrhage, given the underlying acquired hypercoagulability.
\end{abstract}

\section{Keywords}

Bilateral adrenal hemorrhage, Heparin, Splenic infarction, Thrombocytopenia, Thrombosis

\section{Introduction}

Heparin-induced thrombocytopenia (HIT) is a complication of heparin therapy which occurs more commonly in postoperative than medical patients [1]. Up to $50 \%$ of patients undergoing cardiac surgery develop antibodies to platelet factor 4 (PF4) complexed to heparin, attributed to platelet activation and high heparin doses during cardiopulmonary bypass [2]. Only $1 \%$ to $3 \%$ of these patients will develop clinical HIT manifesting with thrombocytopenia with or without thrombosis $[2,3]$. HIT typically presents 5-10 days following exposure to heparin, often with platelet decline greater than $50 \%$ of baseline and is associated with life and limb threatening thrombotic complications. HIT should be suspected in any patient who has received heparin and develops thrombocytopenia, given high mortality (5-10\%) typically a result of thrombotic complications [1].

One increasingly recognized and unusual thrombotic complication seen in HIT is adrenal vein thrombosis with tissue necrosis leading to bilateral adrenal hemorrhage (BAH). This congestive hemorrhage following venous thrombosis should raise high clinical suspicion for HIT. We present a case of splenic vein thrombosis post-cardiac surgery complicated hemorrhagic infarct, reminiscent of that seen in $\mathrm{BAH}$, that led to diagnosis of HIT.

\section{Case Report}

A 62-year-old woman with a history of congestive heart failure, coronary artery disease, diabetes mellitus type 2 , and recent coronary artery bypass graft (CABG) presented to the emergency department with acute weakness, dizziness, and malaise.

Past surgery history was significant for a two-vessel coronary artery bypass graft involving the left internal mammary artery to the left anterior descending artery and the left radial to obtuse marginal artery nine days

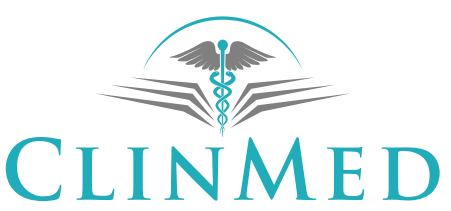

INTERNATIONAL LIBRARY

Citation: Ferry J, Youssef S, WU P, Hegerova L (2020) Heparin-Induced Thrombocytopenia Presenting as Splenic Hemorrhage Following Cardiac Surgery: A Case Report. Int J Blood Res Disord 7:049. doi.org/10.23937/2469-5696/1410049

Accepted: May 23, 2020: Published: May 25, 2020

Copyright: (C) 2020 Ferry J, et al. This is an open-access article distributed under the terms of the Creative Commons Attribution License, which permits unrestricted use, distribution, and reproduction in any medium, provided the original author and source are credited. 
prior to presentation. She had no family history of hematological disorders or hypercoagulability. She was on low-dose aspirin. Her complete blood count (CBC) was normal prior to CABG including platelet count of $268 \times$ $10^{3} / \mu \mathrm{L}$ (normal $150-379 \times 10^{3} / \mu \mathrm{L}$ ) and at hospital discharge was $174 \times 10^{3} / \mu \mathrm{L}$ with post-operative hematocrit of $36 \%$ (normal $37.5 \%-51 \%$ ).

On physical examination she was afebrile, hypotensive with systolic blood pressure in the 70s, and in rapid atrial fibrillation with heart rate 120-130s. She denied abdominal pain and had a benign abdominal examination. Laboratory data revealed white blood cell count $10.7 \times 10^{9} \mathrm{~L}$ (normal 3.4-10.8 $\times 10^{9} \mathrm{~L}$ ), hematocrit 19\%, and platelet count of $49 \times 10^{3} / \mu \mathrm{L}$. Complete metabolic panel and lactate were normal. Troponin was elevated at $0.21 \mathrm{ng} / \mathrm{mL}$ (normal 0.00-0.07 ng/mL.) Chest X-ray was normal. A limited beside echocardiogram demonstrated no pericardial effusion. She received intravenous fluid resuscitation and transferred to the intensive care unit for further evaluation.

Upon arrival to the intensive care unit, she received 2 units of packed red blood cells with appropriate increase to hematocrit $25 \%$ post-transfusion and 1 unit of platelets with increase to $73 \times 10^{3} / \mu \mathrm{L}$. Further laboratory evaluation was negative for hemolysis with normal lactate dehydrogenase $292 \mathrm{U} / \mathrm{L}$ (normal 119-226 $\mathrm{U} / \mathrm{L}$ ) and haptoglobin $98 \mathrm{mg} / \mathrm{dL}$ (normal 34-200 mg/dL). A disseminated intravascular coagulopathy (DIC) panel demonstrated d-dimer of $>40.00 \mu \mathrm{g} / \mathrm{ml}$ (normal $<=0.49$ $\mu \mathrm{g} / \mathrm{ml}$ ), normal fibrinogen $214 \mathrm{mg} / \mathrm{dL}$ (normal 175-475 $\mathrm{mg} / \mathrm{dL}$ ), international normalized ratio INR 1.4 (normal 0.9-1.1), pro time (PT) $16.7 \mathrm{sec}$ (normal 12.0-14.4 sec), partial thromboplastin time (PTT $37.3 \mathrm{sec}$ (normal 22.0-
$35.0 \mathrm{sec}$ ), normal thrombin time (TT), and normal red blood cell morphology.

To evaluate for bleeding, a computed tomography (CT) angiogram of the chest, abdomen and pelvis was ordered and demonstrated filling defects suggestive of right atrial thrombus, splenic vein thrombosis with splenic infarct, and mild to moderate associated hemoperitoneum. She denied abdominal trauma. Surgery service was consulted and concerned about splenic hemorrhage. Hematology was consulted to evaluate etiology of spontaneous splenic vein thrombosis infarct with associated hemorrhagic infarction.

Unfortunately, on day of Hematology evaluation (post-operative day 10) she developed left-sided weakness and balance changes concerning for stroke and $M R I$ revealed infarct involving the distribution of the right anterior cerebral artery. A transesophageal echocardiogram revealed large immobile thrombus in the right atrium with a second thrombus adjacent to this which was mobile and arising from the left tricuspid valve annulus, large thrombus in the left atrial appendage, and absence of a patent foramen ovale (Figure 1). She was not a candidate for thrombolytic therapy due to thrombocytopenia. She was calculated to have a 4Ts score of 7. A heparin PF4 IgG antibody was markedly positive with 3.290 OD. Serotonin release assay was not done due to high probability of HIT. Lower extremity ultrasound was negative for thrombosis while upper extremity ultrasound showed cephalic vein thrombosis. The patient was diagnosed with heparin induced thrombocytopenia with thrombosis (HITT).

Given extensive thrombosis in HIT, anticoagulation

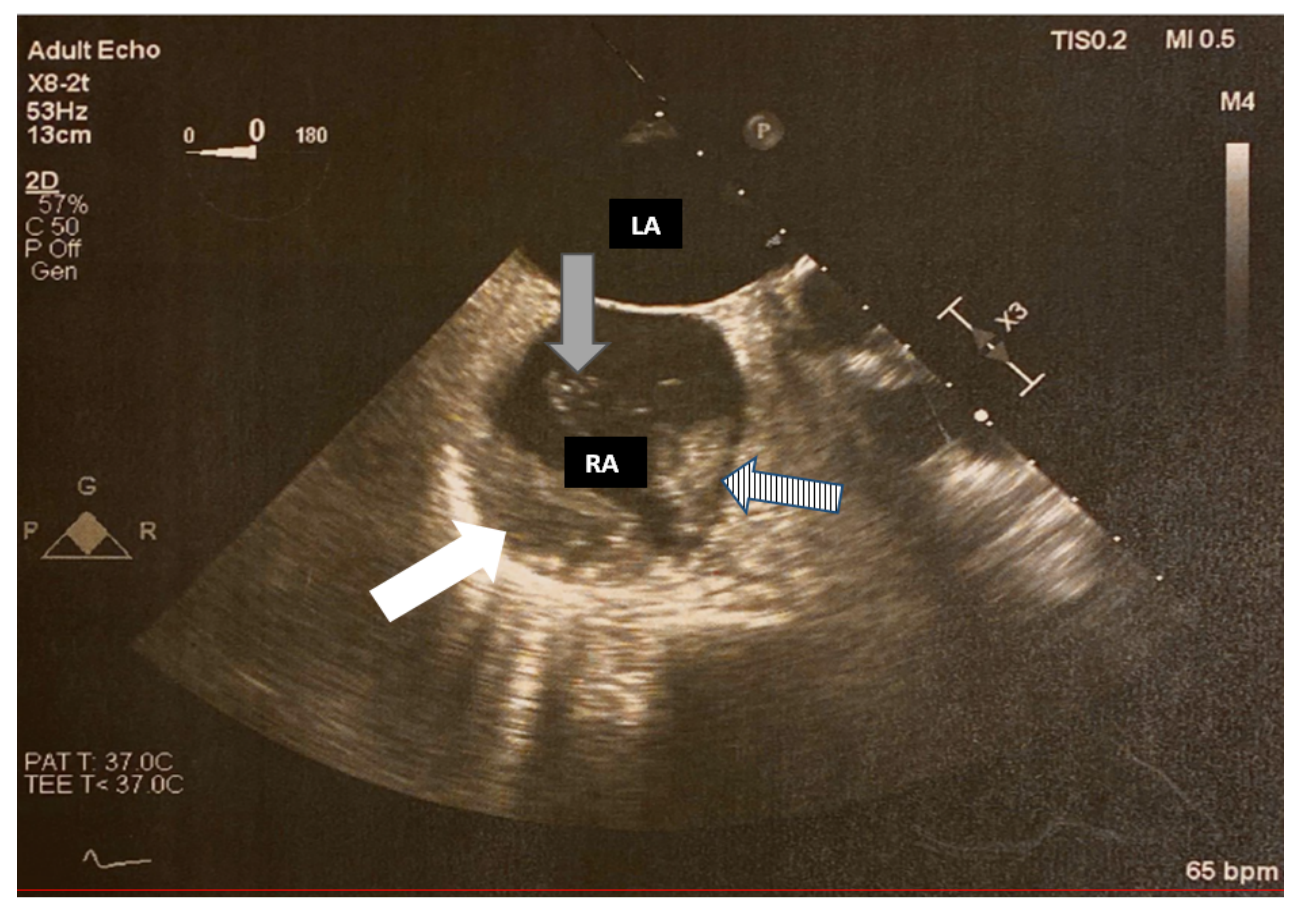

Figure 1: Transesophageal Echocardiogram (TEE). White arrow demonstrating immobile right atrial thrombus. Striped arrow demonstrates second mobile thrombus. Grey arrow demonstrates thrombin strands originating from mobile thrombus. 
was cautiously started with bivalirudin. She remained clinically stable from the intra-abdominal hemorrhage and transitioned to warfarin anticoagulation. Platelets recovered to $108 \times 10^{3} / \mu \mathrm{L}$ four days after starting alternative anticoagulation and greater than $150 \times 10^{3} /$ $\mu \mathrm{L}$ nine days after therapy for HITT initiated. The patient had repeat transthoracic echocardiogram after 3 months of warfarin anticoagulation with resolution of intra-cardiac thrombi. She continues on long-term anticoagulation for atrial fibrillation.

\section{Discussion}

Heparin induced thrombocytopenia (HIT) is a paradoxical prothrombotic syndrome resulting from exposure to heparin-containing products [4-7]. HIT-associated antibodies are frequently detected after cardiac surgery [8]. Thrombosis in the setting of HIT is more commonly associated with the venous system; however arterial thrombus formation is more common in patients receiving heparin for cardiovascular disease [4]. We report a case of atraumatic splenic hemorrhage due to splenic vein infarction as main indicator of the diagnosis of HIT. The presentation is reminiscent of the rare bilateral adrenal hemorrhage due to adrenal vein thrombosis that has been reported in HIT. Given the high mortality of HIT, as high as $28 \%$ in cardiac surgery patients [9], awareness to these unique presentations is critical for earlier intervention.

HIT should be suspected in any patient who has had prior heparin exposure who presents with thrombocytopenia, especially if the fall is greater than $50 \%$ of baseline count and whether the nadir remains within the normal range [5]. Our patient had a high 4T score and HIT PF4 antibody OD of 3.2 and new splenic and atrial thrombosis, confirming a diagnosis of HITT. Anticoagulation was started with parenteral direct thrombin inhibitor and transitioned to warfarin once platelet count was within the normal limit for 2 consecutive days. Alternative anticoagulation is mainstay of therapy for HITT even in setting of hemorrhage, given the underlying acquired hypercoagulability.

A well-documented and increasingly well-recognized thrombotic complication of HIT is bilateral adrenal hemorrhage $(\mathrm{BAH})[6]$. Clues to this rare complication include development of abdominal pain, diarrhea, and fever which often lead to imaging of the abdomen and revelation of injury to the adrenal glands [6] and is confirmed by corticotropin-stimulation test [10]. The risk of $\mathrm{BAH}$ in a case study of HIT by Warkentin, et al. was $1.6 \%$ [11]. Strong arterial blood supply with a single central vein makes the adrenal glands susceptible to congestive hemorrhage following venous thrombosis [12]. We hypothesize that the spleen is at similar risk given it too has a rich arterial supply and single splenic vein.

Literature review reveals few reported cases of splenic vein thrombosis with hemorrhagic infarction as clinical sequelae of HIT. Earlier reports discussed spontaneous splenic ruptures occurring on heparin anticoagulants [13-16] however did not report on HIT antibody testing. One case report had very similar presentation of splenic hematoma after cardiac surgery, with splenectomy pathology showing multiple fibrin thrombi in the spleen [8]. This pathology confirms our hypothesis of microthrombi leading to subsequent hemorrhagic conversion in spleen, as occurs in BAH.

Early imaging to identify these unusual intra-abdominal thrombotic complications of HIT is encouraged. A case report by Lammering, et al. discuss a case of splenic rupture secondary to splenic vein thrombosis resulting from HIT, while the discussion focuses on the utility of multi-detector-row computed tomography in the rapid and accurate diagnosis of HIT related thromboembolic complications [17]. Lammering, et al. describes the case in the setting of non-specific abdominal pain [17] which differed from our patient without abdominal pain.

Treatment of HIT involves alternative non-heparin anticoagulation in accordance with published guidelines including those by the American College of Chest Physicians [1] and the updated American Society of Hematology (ASH) Guidelines for Management of Venous Thromboembolism: Heparin-Induced Thrombocytopenia [18]. Despite hemorrhagic complications such as $\mathrm{BAH}$, or as in our patient intra-abdominal hemorrhage due to splenic infarction, treatment is careful anticoagulation given the underlying etiology for hemorrhage is hypercoagulability. Multiple case reports on $\mathrm{BAH}$ advocate for prompt initiation of appropriate anticoagulation to ensure a successful outcome $[11,19]$. We advocate for similar approach to congestive splenic hemorrhage following splenic vein thrombosis as a consequence of HIT.

This case emphasizes that the diagnosis of HIT should be considered for patients with unusual intra-abdominal thrombosis or hemorrhage, especially within the expected time course following heparin exposure.

\section{Conclusion}

Congestive hemorrhage following venous thrombosis, with recent heparin exposure and declining platelet count should raise clinical suspicion of HIT. The diagnosis of HIT-induced splenic vein thrombosis and subsequent hemorrhagic infarct mimics the rare bilateral adrenal hemorrhage due to adrenal vein thrombosis seen in HIT. Multi detector-row computed tomography should be considered for evaluation of thromboembolic sequelae in patients with unexplained anemia or abdominal pain with suspicion of HITT to exclude adrenal or splenic involvement. Alternative anticoagulation remains the mainstay of therapy.

\section{Disclosure of conflicts of interest}

The authors state that they have no conflict of interest. 


\section{References}

1. Linkins LA, Dans AL, Moores LK, Bona R, Davidson BL, et al. (2012) Treatment and prevention of heparin-induced thrombocytopenia: Antithrombotic therapy and prevention of thrombosis. ( $9^{\text {th }}$ edn), American College of Chest Physicians evidence-based clinical practice guidelines. Chest 141: e495S-e530S.

2. Lillo-Le Louet A, Boutouyrie P, Alhenc-Gelas M, Le Beller C, Gautier I, et al. (2004) Diagnostic score for heparin-induced thrombocytopenia after cardiopulmonary bypass. J Thromb Haemost 2: 1882-1888.

3. Salter BS, Weiner MM, Trinh MA, Heller J, Evans AS, et al. (2016) Heparin-induced thrombocytopenia: A comprehensive clinical review. J Am Coll Cardiol 67: 2519-2532.

4. Ahmed I, Majeed A, Powell R (2007) Heparin induced thrombocytopenia: Diagnosis and management update. Postgraduate Medical Journal 83: 575-582.

5. Baglin TP (2001) Heparin induced thrombocytopenia thrombosis (HIT/T) syndrome: Diagnosis and treatment. $J$ Clin Pathol 54: 272-274.

6. Bakaeen FG, Walkes JC, Reardon MJ (2005) Heparin-Induced Thrombocytopenia Associated With Bilateral Adrenal Hemorrhage After Coronary Artery Bypass Surgery. Ann Thorac Surg 79: 1388-1390.

7. Chow VW, Abnousi F, Huddleston JI, Lin LH (2012) Heparin-induced thrombocytopenia after total knee arthroplasty, with subsequent adrenal hemorrhage. J Arthroplasty 27: 1413.

8. Mitchell C, Riley CA, Vahid B (2007) Unusual complication of heparin-induced thrombocytopenia after mitral valve surgery: Spontaneous rupture of spleen. Ann Thorac Surg 83: 1172-1174.

9. Warkentin TE, Greinacher A (2003) Heparin-induced thrombocytopenia and cardiac surgery. Ann Thorac Surg 76: 2121-2131.

10. Rosenberger LH, Smith PW, Sawyer RG, Hanks JB, Ad- ams RB, et al. (2011) Bilateral adrenal hemorrhage: The unrecognized cause of hemodynamic collapse associated with heparin-induced thrombocytopenia. Critical Care Medicine 39: 833-838.

11. Warkentin TE, Kelton JG (1996) A 14-year study of heparin-induced thrombocytopenia. Am J Med 101: 502-507.

12. Saleem N, Khan M, Parveen S, Balavenkatraman A (2016) Bilateral adrenal haemorrhage: A cause of haemodynamic collapse in heparin-induced thrombocytopaenia. BMJ Case Rep.

13. Burg MD, Dallara JJ (2001) Rupture of a previously normal spleen in association with enoxaparin: An unusual cause of shock. J Emerg Med 20: 349-352.

14. Ghobrial MW, Karim M, Mannam S (2002) Spontaneous splenic rupture following the administration of intravenous heparin: Case report and retrospective case review. Am J Hematol 71: 314-317.

15. Pilkey RM, Lawrence MD, Wolfsohn AL, Walley VM (1994) Splenic rupture resulting from acute pancreatitis after cardiac surgery with intra-aortic balloon pumping: Case report. Can J Surg 37: 428-429.

16. Warkentin TE, Safyan EL, Linkins LA (2015) Heparin-induced thrombocytopenia presenting as bilateral adrenal hemorrhages. N Engl J Med 372: 492-494.

17. Lammering JC, Wang DS, Shin LK (2012) Heparin-induced thrombocytopenia (HIT) causing portosplenic, superior mesenteric, and splenic vein thrombosis resulting in splenic rupture and pulmonary emboli formation. Clin Imaging 36: 865-868.

18. Cuker A, Arepally GM, Chong BH, Cines DB, Greinacher A, et al. (2018) American Society of Hematology 2018 guidelines for management of venous thromboembolism: heparin-induced thrombocytopenia. Blood Adv 2: 3360-3392.

19. Kurtz LE, Yang S (2007) Bilateral adrenal hemorrhage associated with heparin induced thrombocytopenia. Am J Hematol 82: 493-494. 